\title{
A Retrospective Study of Glucagon-Like Peptide 1 Receptor Agonists for the Management of Diabetes After Transplantation
}

\author{
Thiyagarajan Thangavelu $\cdot$ Elizabeth Lyden $\cdot$ Vijay Shivaswamy (D)
}

Received: December 27, 2019 / Published online: February 18, 2020

(c) The Author(s) 2020

\begin{abstract}
Introduction: Management of post-transplant diabetes mellitus is challenging; there is a lack of prospective randomized controlled trials for safety and efficacy of antidiabetic medications in solid organ recipients. Glucagon-like peptide 1 receptor agonists (GLP-1RA) are a relatively new class of medications used to manage type 2 diabetes in the general population. They have several benefits besides glycemic control, including weight loss and improved cardiovascular risk. However, they have not been studied extensively in the post-transplant population for safety and efficacy.

Methods: We conducted a retrospective study of patients who had received kidney, liver, or
\end{abstract}

Enhanced Digital Features To view enhanced digital features for this article go to https://doi.org/10.6084/ m9.figshare.11808834.

T. Thangavelu $\cdot$ V. Shivaswamy $(\square)$

Division of Diabetes, Endocrinology and

Metabolism, Department of Internal Medicine,

Nebraska Medical Center, Omaha, NE, USA

e-mail: vshivaswamy@unmc.edu

E. Lyden

Department of Biostatistics, College of Public Health, University of Nebraska Medical Center, Omaha, NE, USA

V. Shivaswamy

VA Nebraska, Western Iowa Health Care System,

Omaha, NE, USA heart transplant, had diabetes either pre- or post-transplant, and were treated with GLP1RA. We identified seven kidney, seven liver, and five heart transplant recipients who had received GLP-1RA. We assessed changes in immunosuppressant levels, rejection episodes, changes in hemoglobin A1c (HbA1c), weight, and body mass index (BMI) while on the GLP1RA. We also looked at changes in insulin dose, other diabetes medications, heart rate, blood pressure, and renal function.

Results: After a mean follow-up period of 12 months, there were no significant changes in tacrolimus (FK506) levels and renal function for the period of GLP-1RA use. At the end of 12 months, the mean drop in weight was $4.86 \mathrm{~kg}$ [95\% CI $-7.79,-1.93]$. The BMI decreased by a mean of $1.63 \mathrm{~kg} / \mathrm{m}^{2}$ at the end of 12 months [95\% CI $-2.53,-0.73]$. HbA1c decreased from baseline by $1.08 \%$ [95\% CI $-1.65,-0.51$ ], $0.96 \%$ [95\% CI - 1.68, - 0.25], and $0.75 \%$ [95\% CI $-1.55,0.05]$ at 3,6 , and 12 months, respectively.

Conclusions: Our data suggest that GLP-1RA do not affect tacrolimus levels or transplant outcomes in solid organ transplant (SOT) recipients in the short term. GLP-1RA also seem to be as effective in SOT recipients for glycemic control and weight loss as in the non-transplant population with diabetes. 
Keywords: GLP-1RA; Glucose; Immunosuppressants; Type 2 diabetes; Transplant

\section{Key Summary Points}

Glucagon-like peptide 1 receptor agonists

(GLP-1RA) are a potential treatment option for management of diabetes that could offset some of the increased cardiovascular risks associated with transplantation

There are only a few studies that have evaluated the safety and efficacy of GLP1RA post transplantation

We hypothesized that GLP-1RA are safe and effective in managing diabetes in the transplant population

We did a retrospective study looking at changes in immunosuppressant levels, $\mathrm{HbA1c}$, and weight with the use of GLP1RA

Our study shows that GLP-1RA are safe and effective in the short term for the management of diabetes in solid organ recipients

\section{INTRODUCTION}

Diabetes is one of the most significant comorbid conditions after solid organ transplantation. It is associated with long-term adverse effects in transplant recipients. Transplant patients may have pre-existing type 2 diabetes mellitus (T2DM) or develop diabetes after transplantation. Post-transplant diabetes mellitus (PTDM) is associated with reduced graft survival and increases cardiovascular disease-related morbidity and mortality in kidney, heart, and liver transplant recipients $[1,2]$. Similarly, pre-existing diabetes increases the risk for graft loss and decreases survival in solid organ transplant (SOT) recipients $[3,4]$.
Management of diabetes after transplant poses specific barriers, such as potential interactions of antidiabetic medications with immunosuppressant medications as well as reduced renal function. In addition, fluid retention, weight gain, lactic acidosis, delayed gastric emptying, and negative effects on bone mass are all potential adverse effects that can affect the health of the transplant recipient.

Insulin is commonly used for the management of diabetes in transplant recipients, starting immediately post-operation when patients are usually on higher doses of steroids [5]. Later, when the patients are on a more stable dose of steroids, other medications that are commonly used in the non-transplant population can be potentially used. However, options for non-insulin medications are limited because of several factors [1]. Metformin poses a risk of lactic acidosis and cannot be used if renal function is reduced. Pioglitazone poses a risk of fluid retention and can worsen bone mineral density. Previous research has shown the safety and efficacy of dipeptidyl peptidase 4 (DPP4) inhibitors in this population [6]. However, the efficacy of DPP4 inhibitors in glycemic control is limited and, in many situations, patients need a second agent.

Glucagon-like peptide 1 receptor agonists (GLP-1RA) are a relatively new class of diabetes medication that are commonly used for the management of T2DM in the non-transplant population. Besides glycemic control, they also have other potential advantages, such as weight loss and improved cardiovascular risk [7]. GLP1RA also carry a lower risk of hypoglycemia because they induce insulin secretion in a glucose-dependent manner. They can be used in patients with reduced renal function, and because GLP-1RA have weight-lowering benefits, they may be particularly useful to counteract the weight gain that typically occurs posttransplant. Furthermore, the reduction in HbA1c with GLP-1RA ranges from $0.8 \%$ to $2 \%$ [8].

GLP-1RA are eliminated by proteolytic degradation. Therefore, their metabolism does not involve CYP- or transporter-mediated drug-drug interactions [9]. Hence, they are theoretically safe because the chances of 
interaction with immunosuppressant medications are low. GLP-1RA are an attractive option for management of post-transplant diabetes, although only a small number of studies evaluated GLP-1RA for this condition [10-13].

In a study with 12 kidney transplant recipients and 12 controls, GLP-1RA infusion lowered fasting plasma glucose, reduced glucagon concentration, and increased first- and secondphase insulin secretion during a hyperglycemic clamp [13]. In another small study, five patients who were treated with liraglutide post kidney transplant had improvement in glycemic control, weight, and had no adverse effects on graft renal function after a mean follow-up of 19 months [10].

GLP-1RA delay gastric emptying and thus can affect the absorption of immunosuppressant medications [14]. Data on the safety of GLP-1RA in the post-transplant population is also limited. In a small case series of five patients, co-administration of liraglutide with tacrolimus post kidney transplant did not clinically affect the trough tacrolimus levels [11].

We hypothesized that GLP-1RA are safe and effective in the management of diabetes after transplantation.

\section{METHODS}

We retrospectively analyzed kidney, heart, or liver transplantation recipients at the University of Nebraska Medical Center (UNMC) who had either pre-existing T2DM or developed PTDM and were subsequently treated with any of the available GLP-1RA: exenatide, liraglutide, dulaglutide, or semaglutide. Inclusion criteria were previous heart, kidney, or liver transplant, history of T2DM or PTDM, and use of any of the aforementioned GLP-1RA post transplantation. Exclusion criteria were age less than 19 years, type 1 diabetes mellitus (T1DM), and GLP-1RA use for less than 3 months.

The providers started their patients on a titrated GLP-1RA dose per the recommended protocol. Some patients did not reach the maximum dose. Baselines values were defined as the last measured values at, or within 1 month prior to, the time of starting GLP-1RA.
Measurements taken included tacrolimus (FK506) levels, estimated glomerular filtration rate (eGFR), weight, body mass index (BMI), total cholesterol (TC), low-density lipoprotein (LDL), heart rate (HR), and liver function tests (LFT). The changes in these parameters were evaluated at 3,6 , and 12 months compared to baseline values using a paired $t$ test. We also looked at all graft rejection episodes and mortality. Results are reported as mean difference (e.g., 3-month value - baseline value) with a $95 \%$ confidence interval of the difference. Descriptive statistics (counts, percentages, means, standard deviations, medians, and ranges) were used to summarize the data.

Three patients had known thyroid nodules. One patient had a previous thyroidectomy and pathology was benign, another patient had a benign biopsy, and the last patient had a biopsy but the results of that biopsy were not available. Two patients had ultrasounds without nodules reported. All other patients had a normal thyroid on physical exam and had no history of thyroid nodules/neoplasms.

The study was approved by UNMC Institutional Review Board (IRB) protocol \# 806-18-EP. Exemption to consent was approved by the IRB, since there was no or minimal risk to subjects because of the retrospective nature of the study. The study conformed with the Helsinki declaration of 1964, as revised in 2013, concerning human and animal rights.

\section{RESULTS}

We identified 19 patients who fulfilled the inclusion and exclusion criteria. Sixteen patients had T2DM pre-transplant and three patients had PTDM. Baseline characteristics are listed in Table 1.

The most common reason for kidney transplant was end-stage kidney disease due to hypertensive nephrosclerosis followed by diabetic nephropathy. Four patients had received a cadaveric transplant and three had received a living donor kidney. The most common reason for heart transplant was ischemic cardiomyopathy. Tacrolimus was used for immunosuppression in 18 out of 19 patients. Only one 
Table 1 Baseline characteristics

\begin{tabular}{|c|c|}
\hline Characteristic & Value \\
\hline Age (years) & $62(48-71)$ \\
\hline \multicolumn{2}{|l|}{ Sex } \\
\hline Male & $12(63.2 \%)$ \\
\hline Female & $7(36.8 \%)$ \\
\hline \multicolumn{2}{|l|}{ Ethnicity } \\
\hline White & $13(68.4 \%)$ \\
\hline Black & $3(15.8 \%)$ \\
\hline Hispanic & $3(15.8 \%)$ \\
\hline \multicolumn{2}{|l|}{ Organ } \\
\hline Kidney & $7(36.8 \%)$ \\
\hline Liver & $7(36.8 \%)$ \\
\hline Heart & $5(26.3 \%)$ \\
\hline Family history of diabetes & $11(57.8 \%)$ \\
\hline Weight (kg) & $104.78(73.5-147.9)$ \\
\hline BMI $\left(\mathrm{kg} / \mathrm{m}^{2}\right)$ & $35.98(26.96-46.78)$ \\
\hline \multicolumn{2}{|l|}{ Diabetes medication } \\
\hline Insulin & 17 \\
\hline Metformin & 3 \\
\hline DPP4i & 3 \\
\hline SGLT2i & 2 \\
\hline Sulfonylurea & 2 \\
\hline HbAlc (\%) & $8.0(4.6-10.8)$ \\
\hline
\end{tabular}

Data are presented as median (range) or count (percentage)

DPP4i dipeptidyl peptidase 4 inhibitor, $S G L T 2 i$ sodium/ glucose cotransporter 2 inhibitor

patient in our cohort was on cyclosporine. All heart and kidney transplant recipients were on mycophenolate, except for two kidney recipients because of BK viremia or BK virus-associated nephropathy. One of the liver transplant patients was on sirolimus. Five out of seven kidney transplant recipients were on maintenance steroids. One of the liver transplant recipients was on $1 \mathrm{mg}$ of prednisone for systemic lupus erythematosus (SLE), otherwise all other liver and heart transplant recipients were not receiving steroids.

For management of diabetes, 17 patients were on insulin, out of which 15 were on basal-bolus regimen, one was on basal insulin only, and one was on prandial insulin only. Sixteen patients were on insulin analogues (glargine, lispro, and aspart) and one patient was on human insulin-U500 concentration. Five patients were on insulin in combination with oral medications. The mean total daily dose (TDD) of insulin at baseline was 77.11 units. At baseline, two patients were on oral medications only for diabetes management. Metformin and DPP4i were the most commonly used oral medications, followed by sulfonylurea and sodium/glucose cotransporter 2 inhibitors (SGLT2i). The most commonly used GLP-1RA was liraglutide (ten patients), followed by dulaglutide (five patients), and exenatide and semaglutide (two patients each). GLP-1RA were started after a median of 60 months after transplant.

FK506 levels did not show any significant change from baseline throughout the study period. There was no unexpected dose change of immunosuppressant medications during the study period. There was no significant change in renal function measured as eGFR during the 12 months of follow-up. There were no significant changes in LFTs. All safety endpoints are listed in Table 2. There was one episode of rejection and mortality-a heart transplant recipient had acute rejection at 3 months after starting GLP-1RA, had multi-organ failure, and died. The authors are not able to decide if the rejection episode is related to GLP-1RA or not.

Primary and secondary efficacy endpoints are listed in Table 3 . There was a statistically significant decrease in the mean weight and BMI between baseline and 3, 6, and 12 months. At the end of 12 months, there was mean drop in weight by $4.86 \mathrm{~kg}$ [95\% CI - 7.79, - 1.93] and BMI decreased by a mean of $1.63 \mathrm{~kg} / \mathrm{m}^{2}$ [95\% CI - 2.53, - 0.73]. HbA1c decreased from baseline by $1.08 \%$ [95\% CI - 1.65, - 0.51], $0.96 \%$ [95\% CI - 1.68, - 0.25], and 0.75\% [95\% CI $-1.55,0.05]$ at 3,6 , and 12 months, respectively. The mean TDD of insulin 
Table 2 Change from baseline of tacrolimus and renal function

\begin{tabular}{lcll}
\hline Variables & $\begin{array}{l}\text { Change from baseline } \\
\text { to } \mathbf{3} \text { months }\end{array}$ & $\begin{array}{l}\text { Change from baseline } \\
\text { to } \mathbf{6} \text { months }\end{array}$ & $\begin{array}{l}\text { Change from baseline } \\
\text { to 12 } \text { months }\end{array}$ \\
\hline FK506 $(\mathrm{ng} / \mathrm{mL})$ & $0.36(-1.16,1.88)$ & $0.27(-0.98,1.53)$ & $0.82(-0.73,2.36)$ \\
eGFR $\left(\mathrm{mL} / \mathrm{min} / 1.73 \mathrm{~m}^{2}\right)$ & $-0.19(-3.55,3.18)$ & $1.38(-0.76,3.51)$ & $1.08(-1.63,3.80)$ \\
HR $(\mathrm{bpm})$ & $6.63(2.31,10.96)$ & $4.94(-0.47,10.36)$ & $3.62(-6.31,13.54)$ \\
\hline
\end{tabular}

Data are presented as mean $(95 \% \mathrm{CI})$

$e G F R$ estimated glomerular filtration rate, $H R$ heart rate

Table 3 Change of efficacy endpoints from baseline

\begin{tabular}{llcl}
\hline Variables & $\begin{array}{l}\text { Change from baseline } \\
\text { to } 3 \text { months }\end{array}$ & $\begin{array}{l}\text { Change from baseline } \\
\text { to } \mathbf{6} \text { months }\end{array}$ & $\begin{array}{l}\text { Change from baseline } \\
\text { to 12 months }\end{array}$ \\
\hline Weight $(\mathrm{kg})$ & $-2.42(-3.88,-0.96)$ & $-2.84(-4.94,-0.74)$ & $-4.86(-7.79,-1.93)$ \\
BMI $\left(\mathrm{kg} / \mathrm{m}^{2}\right)$ & $-0.89(-1.44,-0.33)$ & $-1.07(-1.85,-0.29)$ & $-1.63(-2.53,-0.73)$ \\
HbAlc $(\%)$ & $-1.08(-1.65,-0.51)$ & $-0.96(-1.68,-0.25)$ & $-0.75(-1.55,0.05)$ \\
TDD of insulin (units) & $-12.06(-21.95,-2.17)$ & $-14.27(-23.63,-4.91)$ & $-16.25(-33.32,0.82)$ \\
Total cholesterol $(\mathrm{mg} / \mathrm{dl})$ & $-10.45(-32.22,11.31)$ & $-12.00(-41.53,17.53)$ & $-25.88(-44.28,-7.47)$ \\
LDL $(\mathrm{mg} / \mathrm{dl})$ & $-18.64(-38.45,1.18)$ & $7.42(-12.30,11.30)$ & $-20.63(-38.16,-3.09)$ \\
\hline
\end{tabular}

Data are presented as mean $(95 \% \mathrm{CI})$

$B M I$ body mass index, $H b A 1 c$ hemoglobin A1c, $T D D$ total daily dose, $L D L$ low density lipoprotein

decreased from baseline by 12.06 units, 14.27 units, and 16.25 units at 3, 6, and 12 months, respectively. More than half the patients $(57 \%)$ were able to decrease insulin requirements 1 year after starting GLP-1RA. Figure 1 shows the spaghetti plot of individual variables for all subjects at different time points.

Two patients who were on combination of insulin and oral medications stopped oral medications after starting GLP-1RA. The use of oral medications decreased from $37 \%$ to $15 \%$. Overall, $57 \%$ of patients were able to decrease the number of oral medications.

The most reported side effect was nausea in five patients. Three patients stopped the medication because of gastrointestinal (GI)-related adverse effects and two stopped because of cost (not covered by insurance or high co-pay). There were no reported incidents of severe hypoglycemia, pancreatitis, or malignancy, including thyroid cancers, during the follow-up period. There was a tendency towards an increase in heart rate, which was only statistically significant at 3 months. Heart rate increased by $6.6 \mathrm{bpm}$ at 3 months and increased by $3.6 \mathrm{bpm}$ at 12 months, but the increase at 12 months was not statistically significant.

\section{DISCUSSION}

In SOT recipients, the choice of medication to treat comorbid conditions is significantly dependent on the interactions of drugs with immunosuppressants and other potential adverse effects that compromise the graft function. GLP-1RA can potentially slow gastric emptying and delay absorption. All subjects in our study were followed by our renal, cardiac, and liver transplant teams and had complete data on safety measures of FK506 levels, rejection episodes, medication changes, renal function, transaminases, and adverse effects. There were no differences in FK506 levels while on 

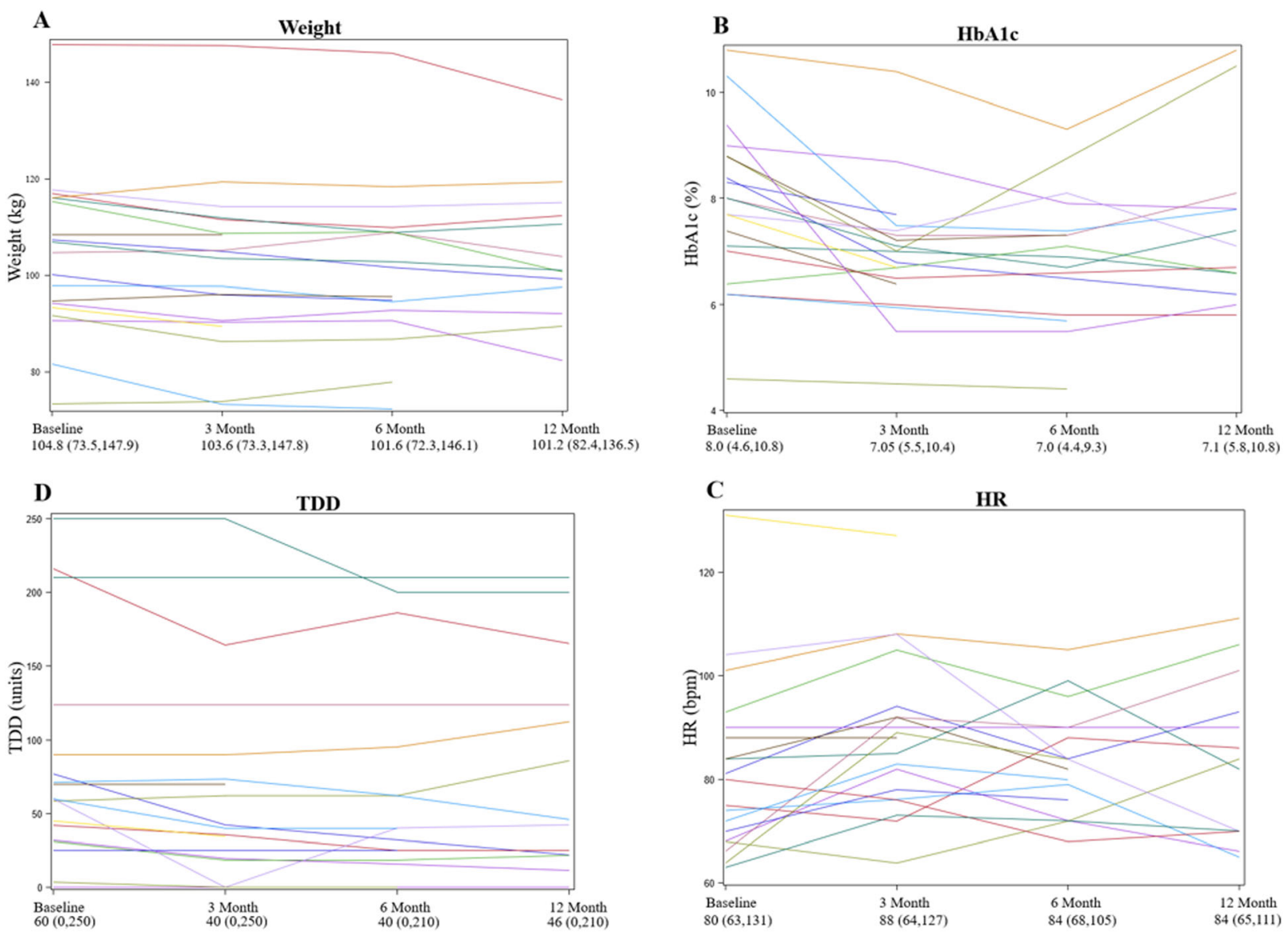

Fig. 1 Spaghetti plot of a weight, b HbAlc, c HR, and d TDD of all subjects from baseline to 12 months. Median and range are given at each time point. $H R$ heart rate, $T D D$ total daily dose of insulin, $b p m$ beats per minute

GLP-1RA in our cohort. Our results are similar to those of Pinelli et al. [11], who reported a small case series of five patients that received co-administration of liraglutide with tacrolimus post kidney transplant; liraglutide did not clinically affect the trough tacrolimus levels. Singh et al. [12] also reported in their study of dulaglutide in SOT recipients that the immunosuppressant regimen remained stable without any increased dose adjustment. This is in contrast to the Liou et al. [10] study of five patients on liraglutide after kidney transplant, who reported that three patients could reduce the dose of tacrolimus and maintain optimal therapeutic levels. Renal function was also not affected in our cohort during the GLP1RA use. The most common side effect to GLP1RA reported in our study was nausea, which is commonly reported in the population of non- transplant patients who also take this class of medication. There were no other serious adverse effects noted in our study.

Our data shows that GLP-1RA improved glycemic control, with improvements in HbA1c $(-0.75 \%)$ and decreases in total daily dose of insulin (16.25 units) 12 months from baseline in SOT recipients. The number of oral medications decreased with GLP-1RA initiation, similar to results reported by Singh et al. [12]. We saw reductions in weight and BMI in our cohort by a mean of $4.86 \mathrm{~kg}$ and $1.63 \mathrm{~kg} / \mathrm{m}^{2}$, respectively, at the end of 12 months. Our results in weight and BMI reduction are higher than what has been previously reported by Singh et al.; however, this may be because the baseline BMI in our cohort was higher than the cohort in Singh et al. 
The important difference in our study compared to earlier studies mentioned is that our cohort was on different GLP-1RA and not a particular agent, e.g., either dulaglutide or liraglutide. Our cohort also included an almost equal number of kidney, heart, and liver transplant recipients.

The current study was retrospective and thus there was no control group. In addition, the baseline values are single measurements prior to start of GLP-1RA, which may not reflect the true status prior to GLP-1RA start. Our cohort size is small and included three different organ transplant recipients and consequently different immunosuppressive regimens. This could have resulted in different effects on glucose metabolism. However, our cohort was on a stable immunosuppressive regimen and GLP-1RA was started after a mean of 60 months after the transplant. Therefore, these effects would have stayed the same or changed only minimally from before to after starting the GLP-1RA. Our study is unique as it is the first study to report on the use of multiple GLP-1RA in the transplant population.

\section{CONCLUSIONS}

Our study adds to the growing body of literature that the currently available GLP-1RA can be used for the management of post-transplant diabetes without affecting tacrolimus levels or transplant outcomes. Furthermore, SOT recipients taking GLP-1RA can expect similar glycemic and weight reduction effects as in the non-transplant population with diabetes.

\section{ACKNOWLEDGEMENTS}

We thank Dr. Clifford Miles, Dr. Timothy McCashland, Dr. Adam Burdorf, and Dr. Brian Boerner for their invaluable help in identifying the subjects for the study.

Funding. No funding or sponsorship was received for this study or publication of this article.
Authorship. All named authors meet the International Committee of Medical Journal Editors (ICMJE) criteria for authorship for this article, take responsibility for the integrity of the work as a whole, and have given their approval for this version to be published.

Disclosures. Thiyagarajan Thangavelu, Elizabeth Lyden, and Vijay Shivaswamy have nothing to disclose.

Compliance with Ethical Guidelines. The study was approved by UNMC Institutional Review Board (IRB) protocol \# 806-18-EP. Exemption to consent was approved by the IRB, since there was no or minimal risk to subjects because of the retrospective nature of the study. The study conformed with the Helsinki declaration of 1964, as revised in 2013, concerning human and animal rights.

Open Access. This article is licensed under a Creative Commons Attribution-NonCommercial 4.0 International License, which permits any non-commercial use, sharing, adaptation, distribution and reproduction in any medium or format, as long as you give appropriate credit to the original author(s) and the source, provide a link to the Creative Commons licence, and indicate if changes were made. The images or other third party material in this article are included in the article's Creative Commons licence, unless indicated otherwise in a credit line to the material. If material is not included in the article's Creative Commons licence and your intended use is not permitted by statutory regulation or exceeds the permitted use, you will need to obtain permission directly from the copyright holder. To view a copy of this licence, visit http://creativecommons.org/licenses/by$\mathrm{nc} / 4.0 /$.

\section{REFERENCES}

1. Shivaswamy V, Boerner B, Larsen J. Post-transplant diabetes mellitus: causes, treatment, and impact on outcomes. Endocr Rev. 2016;37:37-61. 
2. Jenssen T, Hartmann A. Post-transplant diabetes mellitus in patients with solid organ transplants. Nat Rev Endocrinol. 2019;15:172-88.

3. Gaynor JJ, Ciancio G, Guerra G, et al. Single-centre study of 628 adult, primary kidney transplant recipients showing no unfavourable effect of newonset diabetes after transplant. Diabetologia. 2015;58:334-45.

4. Lim WH, Wong G, Pilmore HL, McDonald SP, Chadban SJ. Long-term outcomes of kidney transplantation in people with type 2 diabetes: a population cohort study. Lancet Diabetes Endocrinol. 2017;5:26-33.

5. Thomas MC, Mathew TH, Russ GR, Rao MM, Moran J. Early peri-operative glycaemic control and allograft rejection in patients with diabetes mellitus: a pilot study. Transplantation. 2001;72:1321-4.

6. Boerner BP, Miles CD, Shivaswamy V. Efficacy and safety of sitagliptin for the treatment of new-onset diabetes after renal transplantation. Int J Endocrinol. 2014;2014:617638.

7. Marso SP, Daniels GH, Brown-Frandsen K, et al. Liraglutide and cardiovascular outcomes in type 2 diabetes. N Engl J Med. 2016;375:311-22.

8. Sadhu AR, Schwartz SS, Herman ME. The rationale for use of incretins in the management of new onset diabetes after transplantation (NODAT). Endocr Pract. 2015;21:814-22.
9. Vanhove T, Remijsen Q, Kuypers D, Gillard P. Drugdrug interactions between immunosuppressants and antidiabetic drugs in the treatment of posttransplant diabetes mellitus. Transplant Rev (Orlando). 2017;31:69-77.

10. Liou JH, Liu YM, Chen CH. Management of diabetes mellitus with glucagonlike peptide-1 agonist liraglutide in renal transplant recipients: a retrospective study. Transplant Proc. 2018;50:2502-5.

11. Pinelli NR, Patel A, Salinitri FD. Coadministration of liraglutide with tacrolimus in kidney transplant recipients: a case series. Diabetes Care. 2013;36: e171-2.

12. Singh P, Pesavento TE, Washburn K, Walsh D, Meng S. Largest single-centre experience of dulaglutide for management of diabetes mellitus in solid organ transplant recipients. Diabetes Obes Metab. 2018. https://doi.org/10.1111/dom.13619.

13. Halden TA, Egeland EJ, Asberg A, et al. GLP-1 restores altered insulin and glucagon secretion in posttransplantation diabetes. Diabetes Care. 2016;39:617-24.

14. Uccellatore A, Genovese S, Dicembrini I, Mannucci E, Ceriello A. Comparison review of short-acting and long-acting glucagon-like peptide-1 receptor agonists. Diabetes Ther. 2015;6:239-56. 